\title{
Low Energy STEM of Multi-Layers and Dopant Profiles
}

\author{
P.G. Merli*, A. Migliori*,V. Morandi*, S. Tundo** \\ *CNR-IMM Sezione di Bologna, Via Gobetti 101, 40129 Bologna, Italy \\ **CNR-IMM Sezione di Lecce, Via per Arnesano, 73100 Lecce, Italy
}

Scanning Transmission Electron Microscopy (STEM) is usually associated to incoherent imaging with High Angle Annular Dark Field (HAADF) detector at high energy (100 - $300 \mathrm{keV})$. This operating mode allows atomic resolution $\mathrm{Z}$ contrast images of crystalline materials that, associated with localized energy loss analysis and ab initio theoretical investigations, provides a significant contribute to the understanding of material properties [1].

The STEM investigation at low energy $(\leq 30 \mathrm{keV})$ represents a complementary approach in the examination of material producing a compositional (or mass-thickness) contrast that, in Bright Field (BF) imaging, increases reducing the beam energy [2]. Interesting experiments can be performed with a conventional Scanning Electron Microscope (SEM) equipped with a converter of transmitted electrons in secondary (SE) ones and collecting them by the Everhart-Thornley detector [3] (Fig.1).

The observation of a suitable specimen, consisting of AlAs layers having a thickness of 40,20,10,8,5,3,2,1 nm and 2 and 1 monolayers, separated by GaAs sheets having a constant thickness of $100 \mathrm{~nm}$ (inset of Fig.2) allows interesting conclusions about the performance of the method. Fig. 2 shows a scan line profile of a BF image of a cross-sectioned thinned specimen having a thickness of about $200 \mathrm{~nm}$. It has been obtained using a FEI XL30 SEM equipped with a $\mathrm{LaB}_{6}$ tip, operating at 30 $\mathrm{keV}$ with a spot size of about $6 \mathrm{~nm}$ and a converter collection angle of $5^{\circ}$. The layers of AlAs having a mean atomic number smaller than the GaAs ones appear brighter and clearly visible up to a size of $1 \mathrm{~nm}$, independently of the beam broadening that, for the reported energy and specimen thickness, is about $100 \mathrm{~nm}$. The image layer size results equal to the convolution of the probe size with the layer width. Then a local variation in the composition for a detail crossing the whole specimen can be described with a resolution defined by the dimension of the beam spot.

An interesting application of this investigation method can be the observation of dopants in cross sectioned implanted specimen since the image profile should result from the convolution of the dopant profile with the spot size [3,4]. Fig.3 shows the As profile in Si implanted, at an energy of 5 $\mathrm{keV}$ with a dose of $2 \times 10^{15}$ atoms $/ \mathrm{cm}^{2}$, deduced from numerical simulations [5]. A subsequent thermal annealing at $800{ }^{\circ} \mathrm{C}$ for $30 \mathrm{~min}$ does not significantly affect the dopant distribution. The annealed specimen was cross sectioned, thinned and observed in STEM at low energy using the experimental setup of Fig. 1 with a JEOL 6500 SEM equipped with a Schottky emitter. In Fig. 4a) is reported the scanning profile of a specimen region having a thickness of about $150 \mathrm{~nm}$, acquired at $20 \mathrm{keV}$ using a converter with a collection angle of $9^{\circ}$. Due to the higher atomic number of the dopant the presence of As in the BF image is displayed as a reduction of signal intensity with a position of the minimum and a width that are in agreement with the data reported in Fig. 3. Fig. 4b) shows the simulated image profiles, at the same energy and collection angle, acquired with a Monte Carlo code [3] for different spot sizes: 3,6,10 $\mathrm{nm}$. The comparison of simulated profiles with the experimental one shows that the best accord is achieved for a beam size between $3 \mathrm{~nm}$ and $6 \mathrm{~nm}$. The description of the dopant profile is performed with the same resolution.[6] 


\section{References:}

[1] S.J. Pennycook, Adv. Imag. Elect. Phys. 123 (2002) 173

[2] L. Reimer, Transmission Electron Microscopy, Springer 1983

[3] P.G. Merli et al., Appl. Phys. Lett. 81 (2002) 4535

[4] C.P. Liu et al., Microsc. Microanl. 3 (1997) 352

[5] G. Lulli et al., J. Appl. Phys. 82 (1997) 5958

[6] This research project, code RBAU01M97L, is partially supported by MIUR.

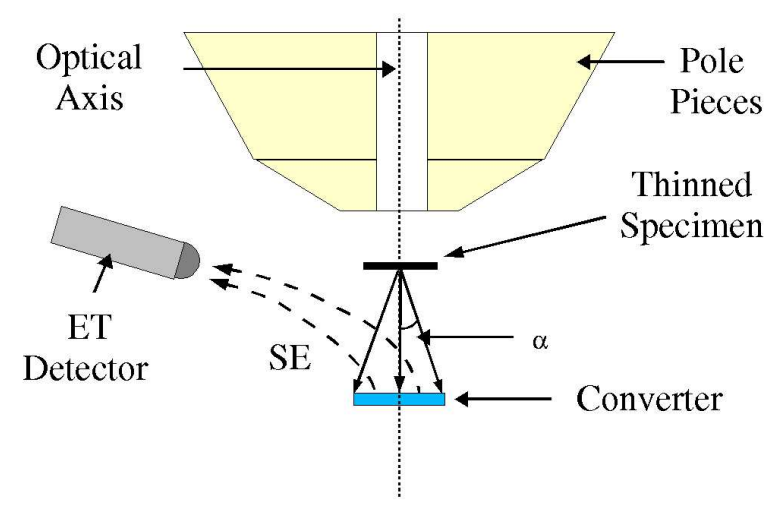

FIG. 1 Scheme of the experimental setup used for BF STEM imaging

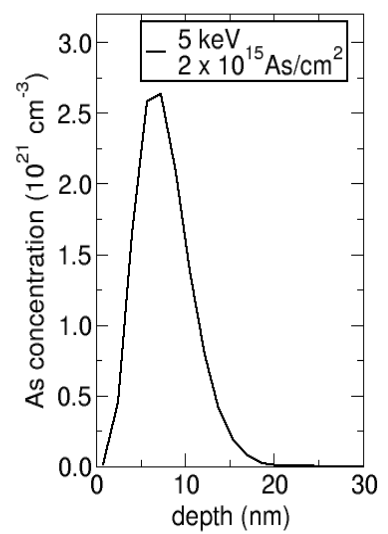

FIG. 3 As concentration versus depth in the implanted silicon specimen.

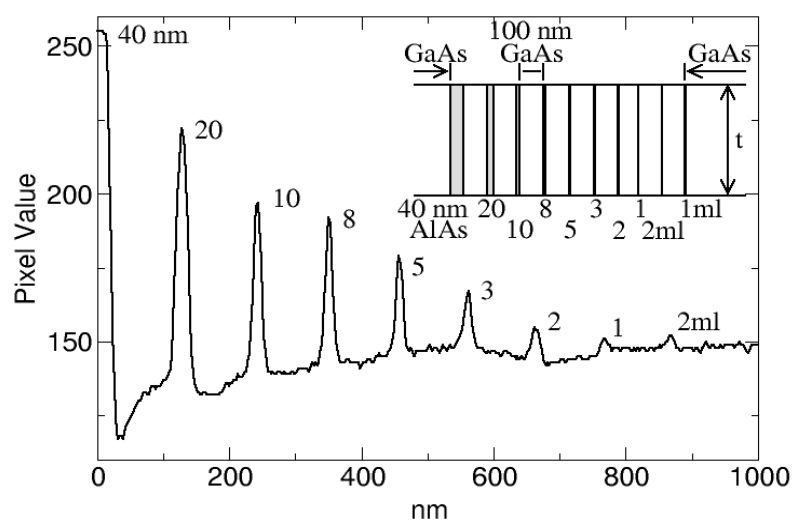

FIG. 2 Experimental SE signal profile for the thinned multilayer shown in the inset. The profile results from the average of 200 scan lines. The signal intensity is indicated in pixel values and ranges from 0 to 255 .
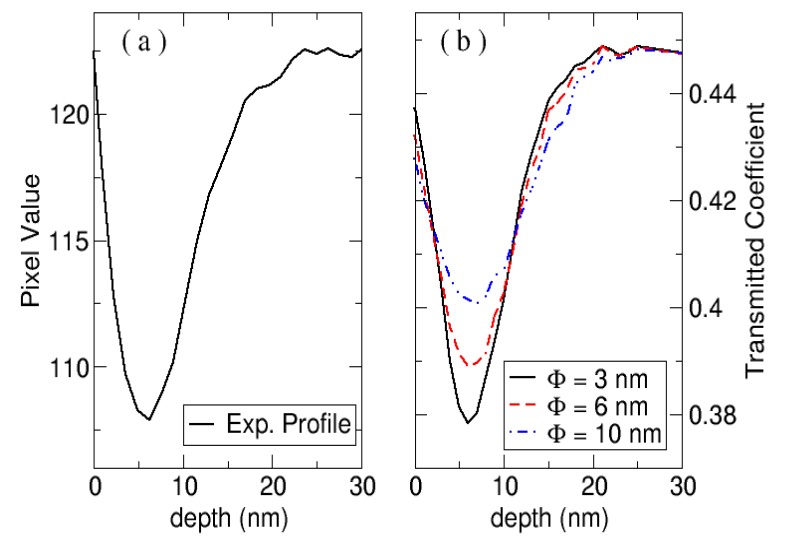

FIG. 4 Experimental, (a) and simulated, (b) SE signal profiles for the implanted specimen having the dopant profile shown in Fig.3. The experimental profile result from the average of 200 scan lines. The signal intensity is indicated in pixel values in (a) while the ordinate of (b) reports the transmission coefficient. 